(C) 2018. This manuscript version is made available under the CC-BY-NC-ND 4.0 license http://creativecommons.org/licenses/by-nc-nd/4.0/

\title{
Locating a Biorefinery in Northern Spain: Decision Making and Economic Consequences
}

\author{
Adrián Serrano-Hernández ${ }^{1}$ and Javier Faulín ${ }^{1}$ \\ (1) Institute of Smart Cities. Public University of Navarre, Pamplona, Spain.
}

\begin{abstract}
:
Biofuels are emerging as a prominent renewable and sustainable energy sources in developed countries. In this sense, this paper presents a case study in which a biorefinery has to be sited is investigated in Northern Spain. Thus, the strategic decision of locating such a facility is deeply investigated through strategic policy evaluation. Then, tactical decisions ranging from purchase policy, transport policy and storage policy are carried out. Only local and limited biomass can be harvested for supplying the biorefinery through a heterogeneous vehicle fleet and two different and mutually exclusive storage strategies are evaluated: direct supply from crops to biorefinery and using intermediate-collectors. Additionally, crop exploitation factors and biorefinery sizes are used to generate several scenarios in which the strategic decision of location as well as all the tactic decisions are made. Some mixed integer linear programming models are proposed to figure out all relevant decision problems.

The results suggest that the northwest study area as the best option to locate the biorefinery and recommend the intermediate-collector storage strategy. Moreover, key information about critical biomass, crops and times are also provided.
\end{abstract}

Keywords: biofuel, biorefinery; mixed integer linear programming; facility location problem; biomass

\section{Introduction}

The consequences of choosing a wrong place to locate a facility may be dire. Appropriate location of industrial plants is particularly important to contribute to economic, social and sustainable objectives, so it should not be superficially done. Therefore, it is required to analyze all alternatives and investigate conditions surrounding them in terms of infrastructure and supply. In that sense, facility location decisions have a strategic nature. Generally, they are made for the long run and involve the whole company. Then, operational and tactics decisions are made based on the strategic infrastructure previously designed. Papadakis and Barwise (2012) developed five characteristics of strategic decisions: (1), they are huge, risky and with long term effects; (2), they 
are a link between thoughtful and emergent strategy; (3), they are a source of company knowledge; (4), they are a critical and challenging step for individual managers; and (5), they are highly multidisciplinary. Thus, a high degree of reflection and judgment by the decision maker is required to deal with such decisions.

Biofuels are considered a promising alternative to conventional fossil fuel in the short and medium term. The European Union is heavily dependent on imported energy resources, especially oil. Actually, $65 \%$ of oil consumption in EU is burnt in the transport sector, which contributes to increase greenhouse gas emissions (European Environment Agency, 2015). According to the same institution, if measures are not taken, the dependence of the EU on imported oil could rise to $90 \%$ by 2020 and Europe will be unable to achieve the goal of reducing emissions of greenhouse gases by $20 \%$ by 2020 . In this context, finding alternative sources of energy for transport is essential to divert oil demand towards less polluting sources. Therefore, encouragement of the use of biofuels in transport (mainly bioethanol and biodiesel) has become a priority in the EU energy policies. Moreover, bioproducts market is constantly expanding as applications in pharmaceutical, chemical, paper, and energy sectors are increasing. The link between biomass and bioproduct is the biorefinery. A biorefinery is the integrated facility in which it is used biomass for the production of bioproduts through thermochemical (combustion, gasification, pyrolysis and/or liquefaction) and biological (fermentation, anaerobic digestion, and/or biologic transesterification) processes. Additional general and technical information about biomass, biorefineries and bioproducts can be found in Aresta et al. (2012).

The Strategic Policy Evaluation aims to determine the effects of strategic decisions on business performance through evaluating several scenarios. In this work, it will be presented a case study in which a biorefinery has to be located in Northern Spain given the available biomass in the area. Based on the strategic decision of location, supply chain is adjusted and tactics decisions of purchase policy, transport policy and storage policy are made. Purchase policy involves the kind of biomass to be bought and the crops they come from. Due to feedstock seasonality, a time factor is included. Transport policy comprises quantities to be transported and the type of vehicle used. Finally, storage policy defines optimal level of stocks. The strategy policy evaluation overview is given in Figure 1. Moreover, two different storage strategies are evaluated: whether having intermediate-collectors or not. For organizational purposes, next section review the related literature to biorefineries and location modelling. Section 3 introduces the detailed problem, 
defines the geographical space, and shows the experimental data. Later, results are presented and discussed. Finally, section 5 gives some concluding remarks.

Figure 1 Strategic Policy Evaluation overview

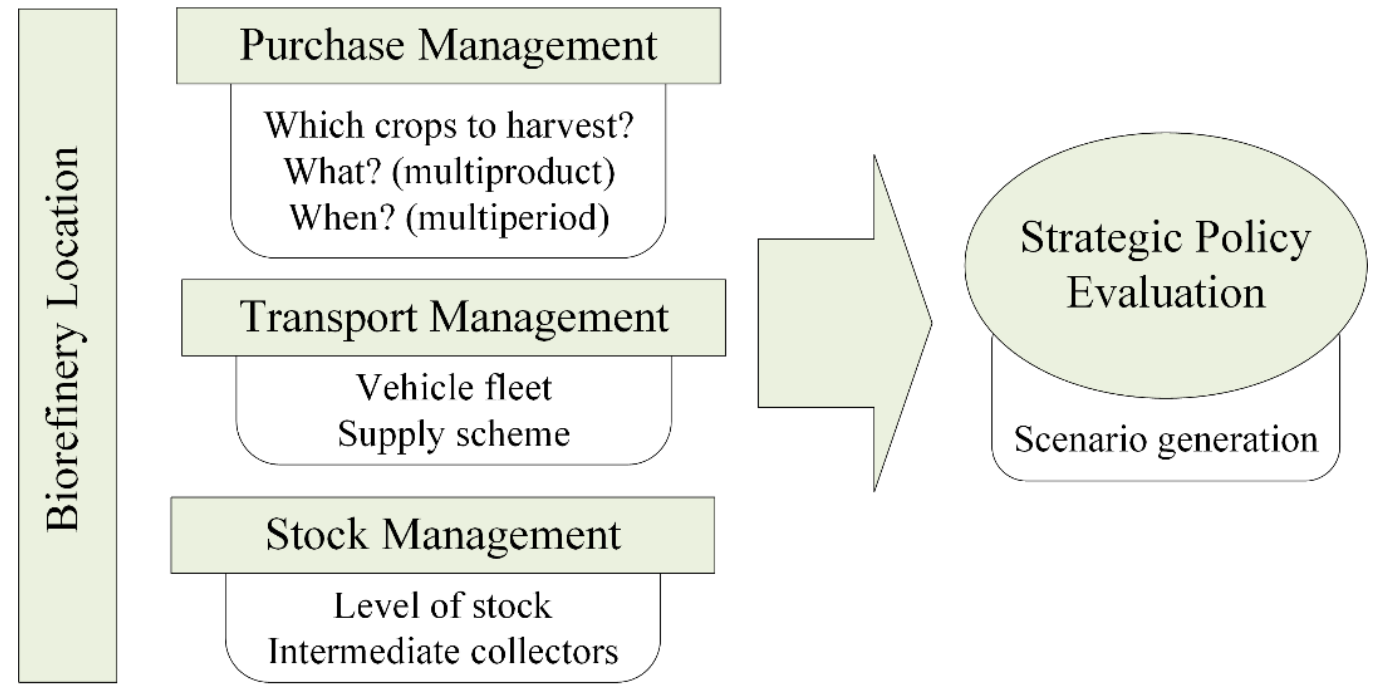

\section{Related literature}

Preliminary works of this paper can be found in Serrano-Hernandez et al. (2015) and SerranoHernandez et al. (2017). In the former, stochasticity of biomass is investigated to determine the biorefinery size. Afterward, the biorefinery is placed accordingly. In the later, a deeper analysis was run: economic and environmental criteria were taken into account to site a biorefinery in Navarre (Spain). Then, purchase management, transport policy and storage planning was optimized. Main differences between those papers and this one are related to the study area, biomass information, model definition and complexity, and conclusions.

Facility location problems are widely studied in the literature. Due to its strategic nature, facility location works are extremely linked to business decisions science. Therefore, those facilities that may be considered significant because their large investment (hotels, huge industrial plants...) or special circumstances (residual wastes, hospitals...) have received attention from the scientific community. Additionally, facility location is highly important for companies that look beyond their country borders and seek for a new place to establish them as observed by Spigarelli and Ly (2016). To do so, they defined the determinants for Chinese companies to expand in Europe, finding that countries with minor rule of law and higher Gross Domestic Product (GDP) per capital are more attractive. In the tourism sector, according to Lado-Sestayo et al. (2016), hotel location is, mainly, the only success factor. They also remarked that credit institutions usually focus on location factors when they have to decide to support a hotel project. Similar conclusions 
were found by Yang et al. (2014) and Masiero et al. (2015). Industrial plant location is further investigated by Ayodele et al. (2016) looking for wind turbine best locations in which they had to care about the wind power in Africa. General information about facility location problems, its role inside the supply chain and sustainability can be found in Zanjirani, and Hekmatfar (2009); Chen et al. (2014) and Melo et al. (2009).

Some works related to biorefinery location can be found in the recent literature. Mainly, those works implement geographical information systems (GIS) in traditional optimization (cost minimization, net present value (NPV) maximization problems), multiobjective optimization, and strategies based on marginal prices. Traditional optimization is investigated by Xie et al. (2009). They aim to develop a tool to support decision making based on GIS to determine the best location of biorefineries. Candidate locations consisted of several points defined beforehand. Then, a mixed integer linear programming (MILP) model is run to minimize transportation cost. Similarly, Marvin et al. (2013) claim that due to important logistic decisions arise (i.e., the location), binary variables should be included. This approach results in MILP models. In this case, the model solves the location and size of several biorefineries as well as their technology and network. Then, the net present value (NPV) chain is optimized within the whole biomass supply chain. Further biomass supply chain characteristics were also investigated in San Miguel et al. (2015). Finally, NPV is again used by Yu et al. (2014).

Interesting research based on multi criteria optimization can be discovered in Mele et al. (2009), You and Wang (2011) and You et al. (2012). Mele et al. (2009) developed a bi-objective MILP in which costs of producing sugar cane as well as its environmental impact are taken into account in an Argentinian region to place energy facilities. A similar study was carried out in Italy (Delivand et al., 2015). Economic (costs) and environmental (greenhouse gas (GHG) emissions) balance is also explored in You and Wang (2011). They developed a multi-period MILP with 49 restrictions to collect the characteristics of the "biomass to liquid" supply chain. Decision variables had to do with the number, size, location and technology of each biorefinery. Thirdly, in the You et al. (2012) work, a three objective problem is presented: environmental (GHG emissions), economic (total annual cost) and social (job creation) criteria. The model simultaneously solves the optimal location and technology of two biorefineries, network design, inventory control, capital investment and other decision variables related to operation management. Epsilon constraint methodology was followed to generate Pareto curves within the three goals.

The change in cost to deliver feedstock as the quantity of required feedstock increases is known as marginal costs (Haque et al. 2014). This concept is widely used to determine the best location for new energy facilities like biorefineries as shown by Panichelli et al (2008). Their proposed methodology can be divided into four steps: 
(1) Create a map of farmland availabilities. The map is divided in $1 \mathrm{~km} \times 1 \mathrm{~km}$ pixels with four pieces of information each: county to which it belongs, the type of soil it has, the proportion of appropriateness for energy crops and the percentage of the county that is suitable for conversion to energy crop.

(2) Calculation of the price. The price of a ton of raw material produced will be equivalent to what the farmer would get with their current settings crops during the life of the biorefinery (a NPV is used to this purpose).

(3) Mapping the cost of a unit of raw material. Using the information of steps 1 and 2, it results in a map with potential biomass supply at each pixel with its price. Then, transport costs are calculated from one pixel to another.

(4) Location of facilities. Potential locations are selected sequentially based on the lower cost previously obtained.

\section{Problem definition}

\subsection{The biorefinery}

A lignocellulosic biorefinery is planned to be placed in northern Spain, covering the regions of Navarre, Aragon and La Rioja. They are leading regions in Spain in renewable energy generation, mainly wind and solar, and they are continuously investing on research and development in order to diversify their energy production. In this sense, bioenergy is seen as a good option to reinforce their leading position. Lignocellulosic biorefineries may use wood, agricultural residues, and energetic crops as biomass. However, due to project characteristics and resource availabilities, just agricultural residues coming from the study region can be used. In a lignocellulosic biorefinery, pentose and hexose saccharides (sugar derived from the biomass) are separated to produce bioethanol and higher value chemicals commodities. Broadly speaking, biorefinery faces a four-hold process, as shown in Figure 2: (1) extracting lignocellulosic material from biomass; (2) decomposing lignocellulosic into cellulose, hemicellulose and lignine; (3) hydrolysis of cellulose and hemicellulose to obtain glucose and xylose; (4) fermentation of glucose and xylose to obtain bioethanol and high value chemical commodities (xylitol and furfural). The reader can find a complete report on lignocellulosic biorefineries in Luo et al. (2010).

Finally, biorefinery size, measured in terms of biomass consumption, is not explicitly optimized, as several size-related scenarios will be considered instead. 
Figure 2 Simplified lignocellulosic biorefinery process, based on Luo et al. (2010)

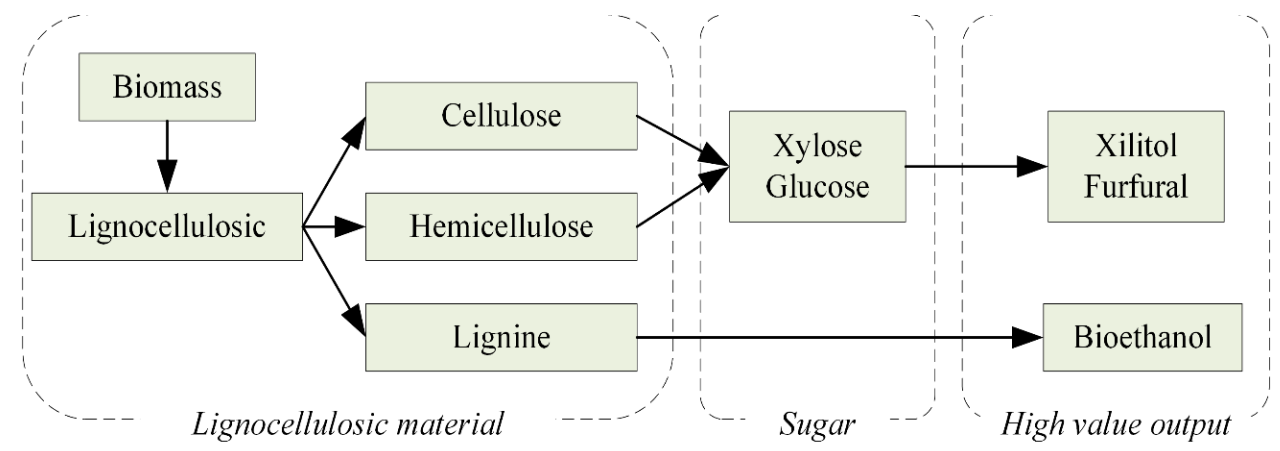

\subsection{The biomass}

Projects based on seasonal natural resources such as biomass are highly geographically dependent. With this respect, availability and density of biomass is investigated focusing on agricultural residues. Note that due to project characteristics only local biomass can be used, i.e. imports are not allowed. Consequently, cereal straw, rice straw, corn straw, rape straw and alfalfa are selected as feedstock to the biorefinery because their wide implementation in the study area (Department of Agriculture of Navarre, 2016; Department of Agriculture of Aragon, 2016; Department of Agriculture of La Rioja, 2016). Winter cereal straws (which include wheat, oat and barley) are the predominant source of biomass in the three regions. They account for about 700,000 annual tons during the previous 15 years. The high seasonality is the main drawback being only available to be harvested during June, July and August. On the other hand, a low humidity rate (around 12\%) and reduced price (around 55-65 €/ton) make cereal a good option. Alfalfa production is about 300,000 tons per year and is available from March to October, but it has higher humidity rate $(60 \%)$ and price $(80-100 € /$ ton). Corn straw is the third most popular biomass in the region with 200,000 tons. It is available in winter time (from November to January), and it has around $25 \%$ humidity with a cost of $65-75 € /$ ton. Finally, rape and rice straws are also taken into account, even though they represent a small share in the total production. Biomass summary is showed in Table 1.

Table 1 Biomass summary available in the study region

\begin{tabular}{r|cccc} 
Biomass & $\begin{array}{c}\text { Availability } \\
\text { (months) }\end{array}$ & $\begin{array}{c}\text { Quantity } \\
\text { ('000 tons) }\end{array}$ & $\begin{array}{c}\text { Humidity } \\
(\%)\end{array}$ & $\begin{array}{c}\text { Price } \\
(€ / \text { ton })\end{array}$ \\
\hline Winter Cereal Straw & June-August & 2,000 & 12 & $55-65$ \\
Corn Straw & Nov- January & 1,250 & 25 & $50-70$ \\
Alfalfa & March- October & 1,000 & 60 & $80-110$ \\
Rape Straw & July- August & 50 & 12 & $70-90$ \\
Rice Straw & October-Nov & 50 & 27 & $55-75$ \\
\hline
\end{tabular}

In order to guarantee sustainability (soil, prices, animal feeding...) an exploitation factor is used in every crop and for each biomass product. It means the proportion of the total resources 
availability that effectively could be used for supplying a biorefinery. Those exploitation factors were carefully chosen conjointly with the Navarrese Agricultural Department based on soil characteristics and current agricultural practice. However, in order to generate several scenarios, the exploitation factor will be thoughtfully analyzed in both cases: an increase and decrease of $50 \%$.

\subsection{The Storage}

Two strategies can be assessed in storage policy. On the one hand, biomass can be unlimitedly stored at the supply point, outside in the countryside. On the other hand, biomass can be transported to a limited-capacity intermediate-collector from crops fields. According to the project characteristics both strategies are mutually exclusive. That is, decision maker has to choose between the direct supply and the possibility of having intermediate collectors. Intermediate-collectors used in this work are rustic warehouses placed in the countryside. They have a 15,000 tons capacity in a 2,400 square meters surface. Real market prices, based on company interviews, were used. Consequently, a yearly fix rent which includes insurance and basic upkeep is taken into account. Additionally, a variable handling cost at the intermediate collector is employed.

Direct supply strategy provides a higher flexibility with respect to the vehicles to choose. It means that transportation from crops to the biorefinery can be made with any type of vehicle. Alternatively, intermediate-collector strategy uses a fix assignment of vehicle as they are usually placed in the countryside with a very limited accessibility. With this respect, only small vehicles can reach to intermediate-collectors from crops because they usually are linked by rural roads. If the vehicle is going directly to the potential biorefinery point from the crop, a large vehicle can be used because of the good communications. Finally, only medium size vehicles can departure from the intermediate-collector facilities. Next subsection will describe vehicle characteristics. Difference in biomass depreciation is the critical factor between both strategies. Intermediatecollectors offer a great protection against external agents: wind, rain, humidity and even thieves. Therefore, depreciation rates are significantly lower in the intermediate collectors than in the countryside. Figure 3 shows time dependent depreciation rates, noting that in winter and springtime they are significantly higher due to climate conditions. Figure 3 also shows the depreciation as a result of the transport activity. This information was elaborated based on internal studies carried out by Spanish Agricultural agencies. 
Figure 3 Depreciation rates in countryside, intermediate-collectors and transport in Spain

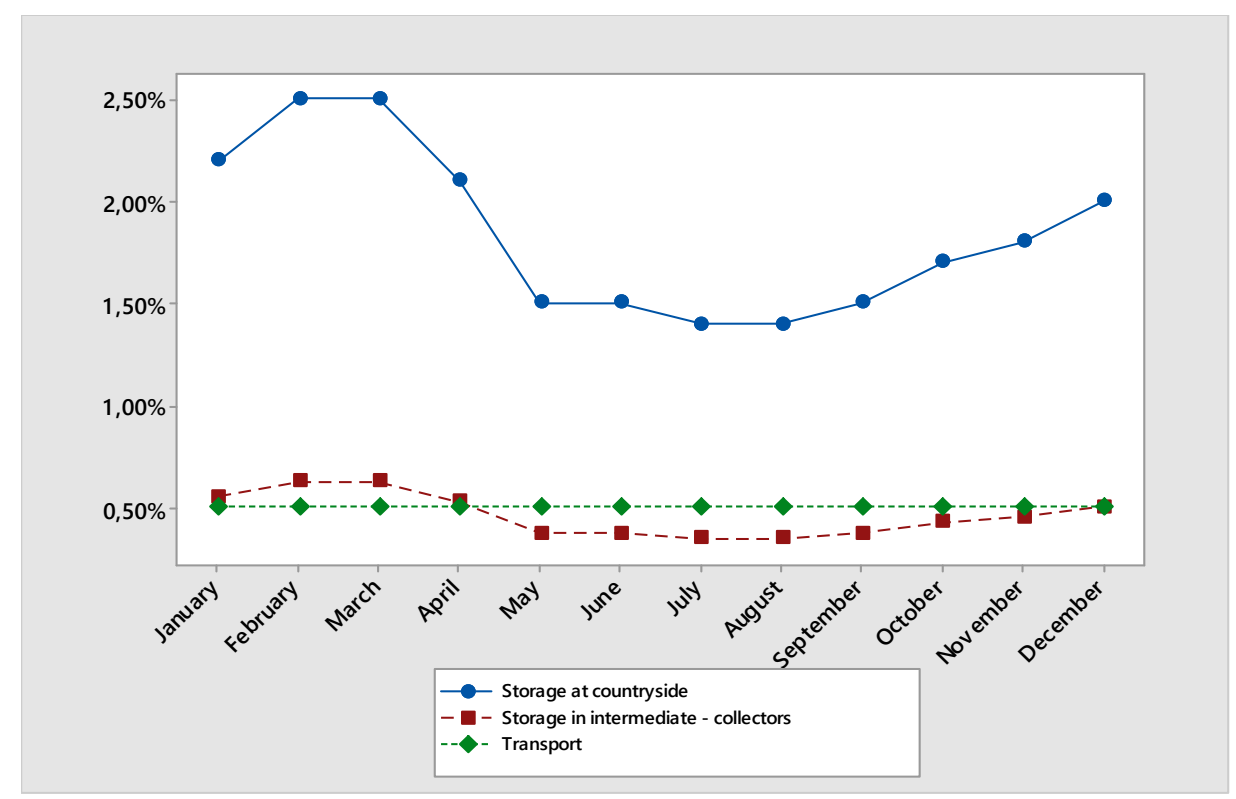

\subsection{The vehicles}

Three types of vehicles are proposed to transport biomass from crops to intermediate-collectors and/or to the biorefinery. Large vehicle (L) is characterized for its higher capacity, being able to transport up to 32 tons. Its huge dimensions make it unappropriated to drive in small roads such as regional or rural ones. Medium vehicle (M) is a traditional truck capable to carry up to 15 tons. Since it is smaller, it is allowed to drive in regional roads but not in rural ones. Finally, small vehicle (S) is a compact and manageable truck, suitable for rural roads. Vehicles characteristics are showed in Table 2.

Table 2 Vehicles Characteristics

\begin{tabular}{l|lll} 
& Vehicle L & Vehicle M & Vehicle S \\
\hline Capacity (tons) & 32 & 15 & 9 \\
Horsepower & 600 & 500 & 160 \\
Axis & 6 & 5 & 2 \\
Allowed in* & HW, NR & HW,NR, ReR & HW, NR, ReR, RuR \\
\hline *HW: Highway; NR: National road; ReR: Regional road; RuR: rural road
\end{tabular}

When the problem faces the direct supply strategy, vehicles are freely selected in the model because crops and potential biorefineries are connected by highways and national roads. However, vehicle characteristics will determine somehow intermediate-collector alternative. Real prices were taken into account based on official estimations (Spanish Government, 2017). Therefore, truck fixed costs and distance dependent cost were carefully added to the model noting that the larger is the vehicle. Thus, the higher fixed costs are, the lower the variable costs are. 


\subsection{The decisions}

A lignocellulosic biorefinery is investigated to be set up in Northern Spain, covering the regions of Navarre, La Rioja and Aragon. The total area accounts for more than 42,000 square kilometers, around $8 \%$ of Spain. Only local and limited biomass (winter cereal straw, corn straw, alfalfa, rape straw, and rice straw) can be harvested for supplying the biorefinery. Two different and mutually exclusive storage strategies have to be assessed:

(1) Direct supply from crops fields to biorefinery. Biomass is, mainly, stored in the countryside with higher depreciation rates. Any kinds of vehicles (L, M, and S) can be used to transport the biomass.

(2) Intermediate-collectors alternative provide a lower depreciation rates. However, an investment on warehouse facilities must be made and lower truck flexibility is considered. Additionally, exploitation factors (the proportion of the total biomass available that effectively could be used for supplying a biorefinery) and biorefinery size (measured as biomass consumption) will generate several scenarios in which the strategic decision of location and all the tactic decisions (purchase policy, transport policy and storage policy) must be taken giving us a reliable strategy policy evaluation.

Figure 4 shows the geographical scope of the problem considered. Firstly, potential locations to host a biorefinery are represented by diamonds. Secondly, triangles stand for potential places to set up intermediate-collectors. Finally, green circles denoted the crops location.

Figure 4 Potential locations (diamonds), intermediate collectors (triangles) and crops fields (circles) located in the decision-making regions in Northern Spain

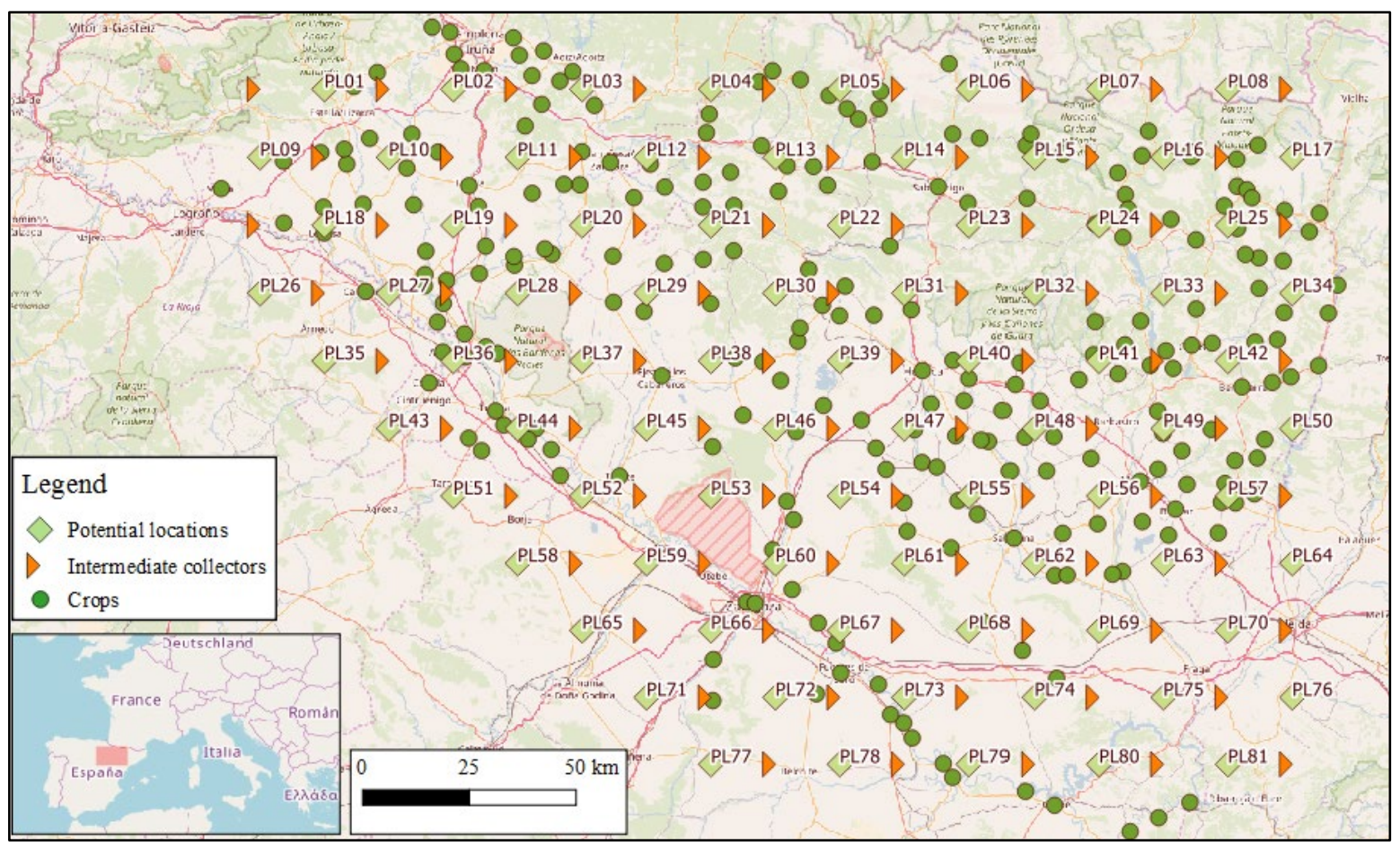




\section{The direct supply model- DSM}

DSM is summarized in the Figure 5. Note that questions in italic correspond to decision variables and capital letters are the key parameters for scenario generation.

Figure 5 Direct supply model overview

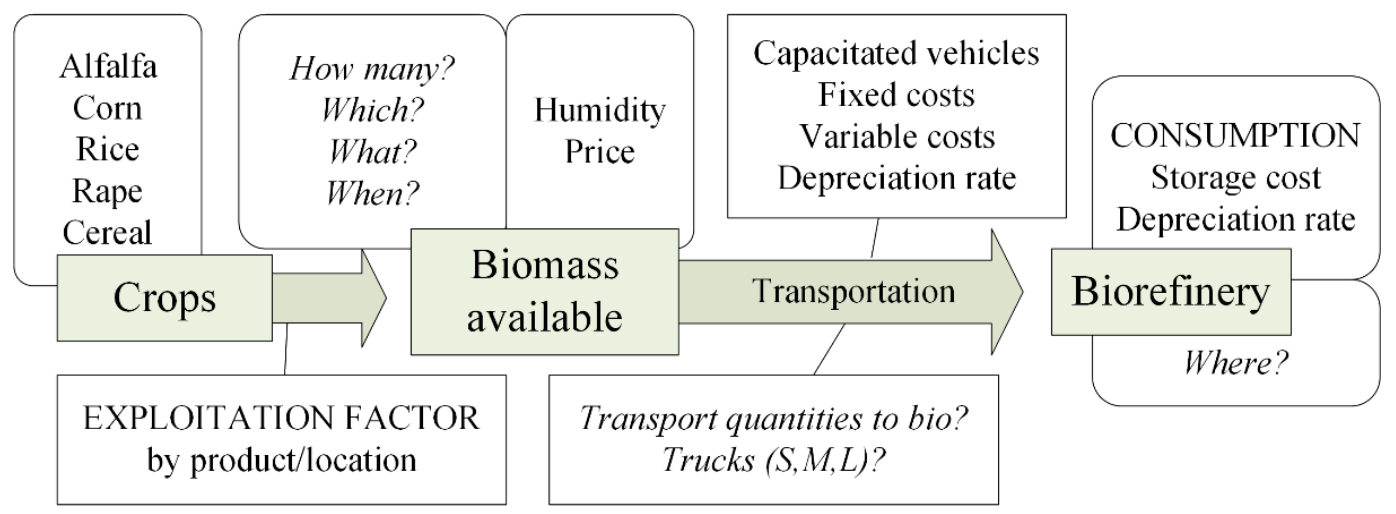

DSM is formulated as mixed integer programming model in which sets, decision variables and parameters are described in Table 3,

Table 4, and Table 5, respectively.

Table 3 Direct supply model sets description

\begin{tabular}{rlc}
\hline Set & Description & Range \\
\hline$I$ & Set of crops fields & $i=1,2 \ldots 354$ \\
$J$ & Set of potential biorefineries & $j=1,2 \ldots 81$ \\
$K$ & Set of vehicles & $k=S, M, L$ \\
$P$ & Set of products & $p=1,2 \ldots 5$ \\
$T$ & Set of months & $t=1,2 \ldots 12$ \\
\hline
\end{tabular}

Table 4 Direct supply model decision variables description

\begin{tabular}{rl}
\hline Variable & Description \\
\hline$X_{j}$ & 1 if the biorefinery is built in potential location $j, 0$ otherwise \\
$V_{i j k t}$ & Number of trucks going from crop $i$ to biorefinery $j$ of type $k$ at time $t$ \\
$B_{i j k p t}$ & Tons of product $p$ bought in crop $i$ at time $t$ to serve potential location $j$ \\
$C_{p i t}$ & Biorefinery $j$ consumption of product $p$ at time $t$ \\
$B S_{j p t}$ & Stock corresponding to potential location $j$ of product $p$ at time $t$ in \\
\hline
\end{tabular}

Table 5 Direct supply model parameter description

\begin{tabular}{rll}
\hline Parameter & Description & Unit \\
\hline$h_{p}$ & humidity of product $p$ & $\%$ \\
$\eta$ & biorefinery monthly consumption & $\mathrm{Tn}$ \\
$\xi_{p t}$ & 1 if product $p$ is available at $t$ & - \\
$d_{i j}$ & distance from crop $i$ to potential location $j$ & $\mathrm{Km}$ \\
$\operatorname{cap}_{k}$ & capacity of vehicle $k$ & Tons \\
$\phi_{p}$ & season duration of product $p$ & Months \\
$\varphi_{p}$ & price of product $p$ & $€$
\end{tabular}




$\begin{array}{rll}\psi_{i p} & \text { total production of } p \text { in } i & \text { Tn } \\ \alpha_{p i} & \text { exploitation factor of product } p \text { in } i & \% \\ F C_{k} & \text { transportation fix cost of vehicle } k & € \\ V C_{k} & \text { transportation variable cost of vehicle } k & € / \mathrm{km} \\ \varsigma & \text { stock cost } & € / \mathrm{Tn} / \mathrm{month} \\ \delta_{t} & \text { losses on stock from time } t \text { to time } t+1 & \% \\ \gamma & \text { losses on transportation } & \%\end{array}$

The DSM is as follows:

$\min$ totalCosts $=$ biomassCosts + transportCosts + storageCosts

$$
\left\{\begin{array}{l}
\text { biomassCosts }=\sum_{i} \sum_{j} \sum_{k} \sum_{p} \sum_{t} \boldsymbol{B}_{\boldsymbol{i j k p t}} \varphi_{p} \\
\text { transportCosts }=\sum_{i} \sum_{j} \sum_{k} \sum_{t} F C_{k} \boldsymbol{V}_{\boldsymbol{i j k t}}+2 V C_{k} \boldsymbol{V}_{\boldsymbol{i j k t}} d_{i j} \\
\text { storageCosts }=\sum_{j} \sum_{p} \sum_{t} B \boldsymbol{S}_{j p t} \varsigma
\end{array}\right.
$$

Subject to,

$\sum_{j} X_{j}=1$

$\sum_{i} \sum_{k} \boldsymbol{B}_{\text {ijpkt }}(1-\gamma)+\boldsymbol{S}_{\text {jpt-1 }}\left(1-\delta_{t}\right)=\frac{\boldsymbol{C}_{\text {pjt }}}{1-h_{p}}+\boldsymbol{B} \boldsymbol{S}_{j p t} ; \forall i \in I, \forall j \in J, \forall p \in P, \forall t \in T$

$\sum_{k} \boldsymbol{B}_{\text {ijpkt }} \leq \psi_{i p} \alpha_{p i} \frac{\xi_{p t}}{\phi_{p}} ; \quad \forall i \in I, \forall j \in J, \forall p \in P, \forall t \in T$

$\sum_{p} \boldsymbol{C}_{\boldsymbol{j p t}}=\boldsymbol{X}_{\boldsymbol{j}} \eta ; \forall j \in J, \forall t \in T$

$\boldsymbol{V}_{\boldsymbol{i j k t}} \geq \sum_{p} \frac{\boldsymbol{B}_{\boldsymbol{i j p k t}}}{\mathrm{cap}_{k}} ; \forall i \in I, \forall j \in J, \forall p \in P, \forall t \in T$

In which the objective function (1) minimizes the total supply chain costs and it is divided into the three considered sources of costs: the costs of purchasing the biomass (1.1), the costs of transporting the biomass (1.2) and the costs of stocking the biomass (1.3).

Constraint (6) determines that one biorefinery must be sited. Constraints (3) describe the intertemporal flows of biomass taking into consideration humidity and depreciation. Constraints (4) state resources availabilities with productions and exploitation factors. Constraints (5) fix the monthly size (consumption) of the biorefinery. Finally, constraints (6) define maximum vehicle capacities.

\section{The intermediate-collector model-ICM}

ICM is described in the Figure 6. As in the previous model, questions in italic correspond to decision variables and capital letters are the key parameters for scenario generation. 
Figure 6 Intermediate-collectors model overview

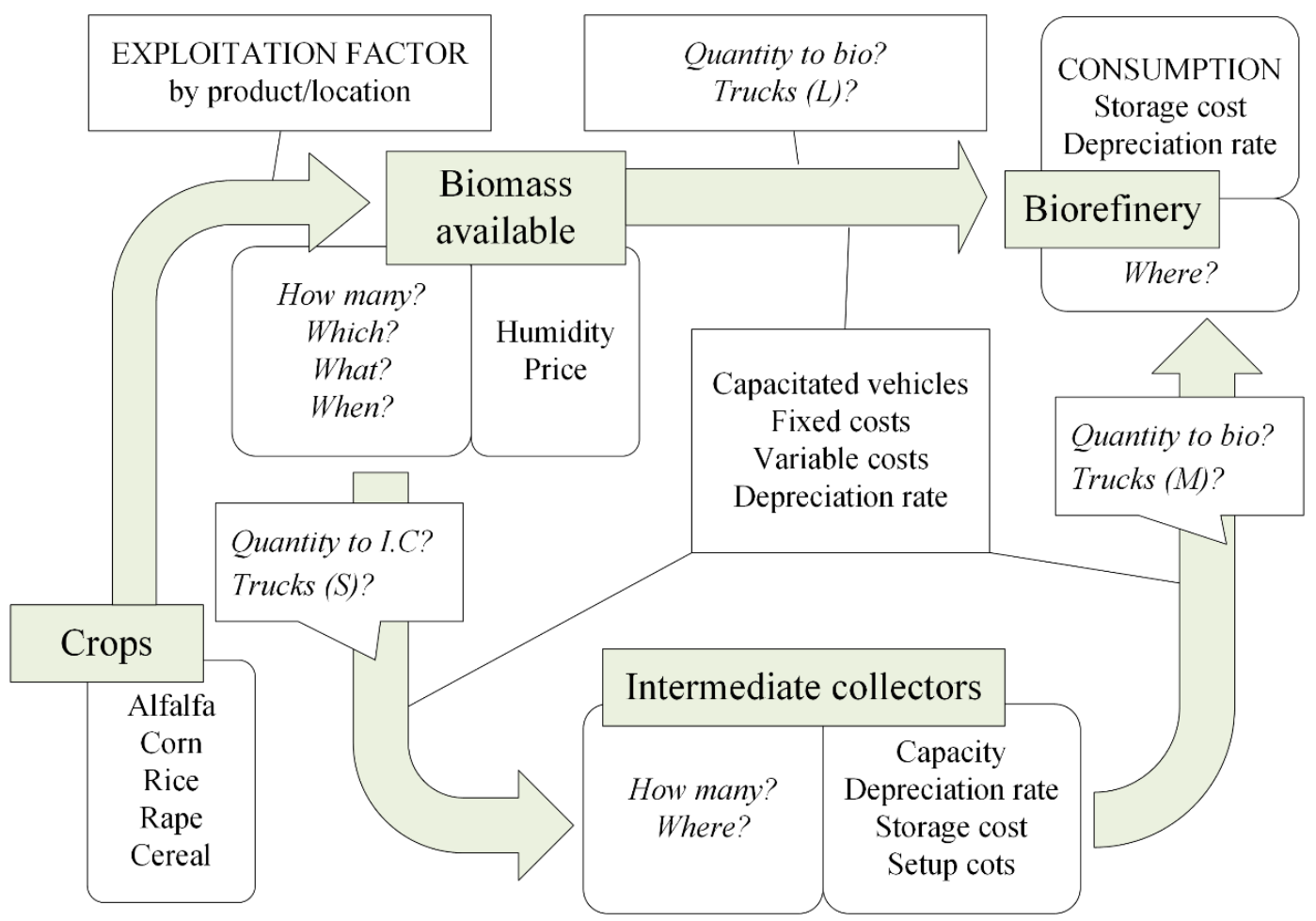

Table 6 Set description

\begin{tabular}{rlc}
\hline Set & Description & Range \\
\hline$I$ & Set of crops fields & $i=1,2 \ldots 354$ \\
$J$ & Set of potential biorefineries & $j=1,2 \ldots 81$ \\
$W$ & Set of intermediate-collector & $w=1,2 \ldots 79$ \\
$P$ & Set of products & $p=1,2 \ldots 5$ \\
$T$ & Set of months & $t=1,2 \ldots 12$ \\
\hline
\end{tabular}

Table 7 Decision variables description

\begin{tabular}{rl}
\hline Variable & Description \\
\hline$X_{j}$ & 1 if the biorefinery is built in potential location $j, 0$ otherwise \\
$Y_{w}$ & 1 if the an intermediate-collector $w$ is set up, 0 otherwise \\
$Q_{i j p t}^{c r o p} \rightarrow$ bio & Tons of product $p$ bought in crop $i$ transported to biorefinery $j$ at time $t$ \\
$Q_{i w p t}^{c r o p \rightarrow I C}$ & Tons of product $p$ bought in crop $i$ transported to intermediate-collector $w$ at time $t$ \\
$Q_{w j p t}^{I C \rightarrow b i o}$ & Tons of product $p$ in intermediate-collector $w$ transported to biorefinery $j$ at time $t$ \\
$V_{i j k t}^{L}$ & Number of large trucks going from crop $i$ to biorefinery $j$ at time $t$ \\
$V_{i w k t}^{S}$ & Number of small trucks going from crop $i$ to intermediate-collector $w$ at time $t$ \\
$V_{w j k t}^{M}$ & Number of medium trucks going from intermediate-collector $w$ to biorefinery $j$ at time $t$ \\
$B_{i p t}$ & Tons of product $p$ bought in crop $i$ at time $t j$ \\
$C_{p i t}$ & Biorefinery $j$ consumption of product $p$ at time $t$ \\
$B S_{j p t}$ & Stock corresponding to potential location $j$ of product $p$ at time $t$ in \\
$C S_{w p t}$ & Stock corresponding to intermediate-collector $w$ of product $p$ at time $t$ in \\
\hline
\end{tabular}


Table 8 Parameter description

\begin{tabular}{|c|c|c|}
\hline Parameter & Description & Unit \\
\hline$h_{p}$ & humidity of product $p$ & $\%$ \\
\hline$\eta$ & biorefinery monthly consumption & $\operatorname{Tn}$ \\
\hline$\beta$ & proportion of consumption which can be stock at the biorefinery & $\%$ \\
\hline$\xi_{p t}$ & 1 if product $p$ is available at $t$ & - \\
\hline$d_{i j}$ & distance from crop $i$ to potential location $j$ & $\mathrm{Km}$ \\
\hline$d_{i w}$ & distance from crop $i$ to intermediate-collector $w$ & $\mathrm{Km}$ \\
\hline$d_{w j}$ & distance from intermediate-collector $w$ to potential location $j$ & $\mathrm{Km}$ \\
\hline $\operatorname{cap}^{L}$ & capacity of a large vehicle & Tons \\
\hline $\operatorname{cap}^{S}$ & capacity of a small vehicle & Tons \\
\hline $\operatorname{cap}^{M}$ & capacity of a medium vehicle & Tons \\
\hline$\phi_{p}$ & season duration of product $p$ & Months \\
\hline$\varphi_{p}$ & price of product $p$ & $€$ \\
\hline$\psi_{i p}$ & total production of $p$ in $i$ & $\operatorname{Tn}$ \\
\hline$\alpha_{p i}$ & exploitation factor of product $p$ in $i$ & $\%$ \\
\hline$F C^{L}$ & transportation fix cost of a large vehicle & $€$ \\
\hline$F C^{S}$ & transportation fix cost of a small vehicle & $€$ \\
\hline$F C^{M}$ & transportation fix cost of a medium vehicle & $€$ \\
\hline$V C^{L}$ & transportation variable cost of a large vehicle & $€ / \mathrm{km}$ \\
\hline$V C^{S}$ & transportation variable cost of a small vehicle & $€ / \mathrm{km}$ \\
\hline$V C^{M}$ & transportation variable cost of a medium vehicle & $€ / \mathrm{km}$ \\
\hline$\varsigma$ & stock cost at biorefinery & $€ /$ Tn/month \\
\hline$\omega$ & cost of setting up an intermediate-collectors & $€$ \\
\hline$\rho$ & capacity of intermediate-collectors & $\operatorname{Tn}$ \\
\hline$\kappa$ & stock cost at intermediate-collector & $€ /$ Tn/month \\
\hline$\delta_{t}$ & losses on stock from time $t$ to time $t+1$ & $\%$ \\
\hline$\gamma$ & losses on transportation & $\%$ \\
\hline
\end{tabular}

The ICM is also formulated as mixed integer programming model in which sets, decision variables and parameters are described in Table 6 , Table 7 , and Table 8 , respectively.

The ICM is as follows:

$$
\begin{aligned}
& \min \text { totalCosts }=\text { biomassCosts }+ \text { transportCosts }+ \text { storageCosts } \\
& \text { biomassCosts }=\sum_{i} \sum_{p} \sum_{t} \boldsymbol{B}_{\text {ipt }} \varphi_{p} \\
& \text { transportCosts }=\left\{\begin{array}{l}
\sum_{i} \sum_{j} \sum_{t} C F^{L} \boldsymbol{V}_{\boldsymbol{i j t}}^{L}+2 C V^{L} \boldsymbol{V}_{\boldsymbol{i j t}}^{L} d_{i j} \\
\sum_{i} \sum_{w} \sum_{t} C F^{S} \boldsymbol{V}_{\boldsymbol{i w t}}^{\boldsymbol{S}}+2 C V^{S} \boldsymbol{V}_{\boldsymbol{i w t}}^{\boldsymbol{S}} d_{i w} \\
\sum_{w} \sum_{j} \sum_{t} C F^{M} \boldsymbol{V}_{\boldsymbol{w j t}}^{\boldsymbol{M}}+2 C V^{M} \boldsymbol{V}_{\boldsymbol{w j t}}^{M} d_{w j}
\end{array}\right. \\
& \text { storageCosts }=\left\{\begin{array}{l}
\sum_{j} \sum_{p} \sum_{t} \boldsymbol{B} \boldsymbol{S}_{\boldsymbol{j p t}} \varsigma \\
\sum_{w} \sum_{p} \sum_{t} \boldsymbol{C} \boldsymbol{S}_{\boldsymbol{w p t}} \kappa+\sum_{w} \boldsymbol{Y}_{\boldsymbol{w}} \omega
\end{array}\right.
\end{aligned}
$$


Subject to,

$$
\begin{aligned}
& \sum_{j} X_{j}=1 \\
& \boldsymbol{B}_{\text {ipt }} \leq \psi_{i p} \alpha_{p i} \frac{\xi_{p t}}{\phi_{p}} ; \forall i \in I, \forall p \in P, \forall t \in T \\
& \boldsymbol{B}_{\text {ipt }}=\sum_{w} \boldsymbol{Q}_{\text {iwpt }}^{\text {crop } \rightarrow I C}+\sum_{j} \boldsymbol{Q}_{\text {ijpt }}^{\text {crop } \rightarrow \text { bio }} ; \forall i \in I, \forall p \in P, \forall t \in T \\
& \sum_{i} \boldsymbol{Q}_{\text {iwpt }}^{\text {crop } \rightarrow I C}(1-\gamma)+\boldsymbol{C S}_{w p t-1}\left(1-\delta_{t}\right)=\sum_{j} \boldsymbol{Q}_{w j p t}^{I C \rightarrow b i o}+C S_{w p t} ; \forall w \in W, \forall p \in P, \forall t \in T \\
& \sum_{i} \boldsymbol{Q}_{i j p t}^{\text {crop } \rightarrow \text { bio }}(1-\gamma)+\sum_{w} \boldsymbol{Q}_{\boldsymbol{w j p t}}^{\boldsymbol{I C} \rightarrow \boldsymbol{b i o}}(1-\gamma)+\boldsymbol{B} \boldsymbol{S}_{\boldsymbol{j p t - 1}}\left(1-\delta_{t}\right)=\frac{C_{p j t}}{1-h_{p}}+\boldsymbol{B} \boldsymbol{S}_{j p t} ; \forall j \in J, \forall p \in P, \forall t \in T \\
& \sum_{p} C_{p i t}=X_{j} \eta ; \forall j \in J, \forall t \in T \\
& \sum_{p} \boldsymbol{B S}_{\boldsymbol{j p t}} \leq \boldsymbol{X}_{\boldsymbol{j}} \beta \eta ; \forall j \in J, \forall t \in T \\
& \sum_{p} C S_{w p t} \leq Y_{w} \rho ; \forall w \in W, \forall t \in T \\
& \boldsymbol{V}_{i j t}^{L} \geq \sum_{p} \frac{\boldsymbol{Q}_{i j p t}^{\text {crop } \rightarrow \text { bio }}}{\text { cap }^{L}} ; \forall i \in I, \forall j \in J, \forall t \in T \\
& \boldsymbol{V}_{\text {iwt }}^{S} \geq \sum_{p} \frac{\boldsymbol{Q}_{\text {iwpt }}^{\text {crop } \rightarrow I C}}{c a p^{S}} ; \forall i \in I, \forall w \in W, \forall t \in T \\
& \boldsymbol{V}_{w j t}^{M} \geq \sum_{p} \frac{\boldsymbol{Q}_{\boldsymbol{w j p t}}^{I C \rightarrow \boldsymbol{b i o}}}{c a p^{M}} ; \forall w \in W, \forall j \in J, \forall t \in T
\end{aligned}
$$

The objective function again minimizes the total costs (7). However, a richer range of costs are considered. Firstly, the costs of purchasing feedstock remains the same as before (7.1). Transportation costs now consider all different alternatives of reaching the biorefinery with a heterogeneous fleet (7.2) to (7.4). Finally, costs of stocking biomass is divided into stocking in the biorefinery main warehouses (7.5) and stocking in the intermediate-collector facilities, taking into account the extra costs of building them (7.6).

Constraint (8) ensures that only one biorefinery has to be set up. Constraints (9) guarantee resources availabilities given the production and exploitation factors. Constraints (10) define biomass from crops fields can go to either the biorefinery or the intermediate-collectors. Constraints (11) and (12) describe the intertemporal flows of biomass from crops fields to intermediate-collectors and the biorefinery. Note that those constraints consider depreciation in transportation and storage as well as the biomass humidity. Constraints (13) determine the monthly consumption of the biorefinery. Finally, constraints (16) to (18) define vehicles utilization. 


\section{Results}

Mathematical models were coded in the General Algebraic Modelling System (GAMS) and solved using CPLEX 14.1. They were run in an INTEL® i5 @2400 with 8 GB RAM. Justification of using the exact method is based on two factors. On the one hand, literature on facility location problems reveals exact method as the common methodology to solve this kind of problems. On the other hand, implementation of heuristic methodologies will not guarantee optimum solutions, and mainly used when exact methods fail. Thus, given the strategic nature of facility location problems it is preferred to obtain the highest quality solution rather than fast ones. For that reason, a time limit of 10 hours was set to each run. That limit was not exceed in any case.

24 scenarios were generated for each strategy (direct supply and intermediate-collector) based on biorefinery size and exploitation factor, as described in Table 9. Biorefinery size analysis ranges from 150,000 net tons of yearly consumption up to 500,000 tons. Those plant capacities are consistent with the total biomass production in the area. Moreover, exploitation factor was analyzed in cases they increase $50 \%$ and they decrease $50 \%$.

Table 9 Scenarios $S_{i}(i=1,2, \ldots, 24)$ based on size and exploitation factor

\begin{tabular}{cccc} 
& \multicolumn{3}{c}{ Exploitation Factor } \\
\hline Size & Base $=1$ & 1.5 & 0.5 \\
\hline 150,000 & $\mathrm{~S} 1$ & $\mathrm{~S} 2$ & $\mathrm{~S} 3$ \\
200,000 & $\mathrm{~S} 4$ & $\mathrm{~S} 5$ & $\mathrm{~S} 6$ \\
250,000 & $\mathrm{~S} 7$ & $\mathrm{~S} 8$ & $\mathrm{~S} 9$ \\
300,000 & $\mathrm{~S} 10$ & $\mathrm{~S} 11$ & $\mathrm{~S} 12$ \\
350,000 & $\mathrm{~S} 13$ & $\mathrm{~S} 14$ & $\mathrm{~S} 15$ \\
400,000 & $\mathrm{~S} 16$ & $\mathrm{~S} 17$ & $\mathrm{~S} 18$ \\
450,000 & $\mathrm{~S} 19$ & $\mathrm{~S} 20$ & $\mathrm{~S} 21$ \\
500,000 & $\mathrm{~S} 22$ & $\mathrm{~S} 23$ & $\mathrm{~S} 24$ \\
\hline
\end{tabular}

Figure 7 shows the optimal emplacement for the biorefinery using either direct supply (DS) or intermediate-collectors (IC). Numbers can be looked up in the Figure 4. Most recurrent location for all alternative is the potential location number 20. However, significant differences arise if we pay attention carefully. According to the results, the Northwest of the study area seems to be an appropriate zone to locate the biorefinery because it accounts for almost all the optimal locations. Potential location 60 got best position three times corresponding to cases in which exploitation factor was extreme. Interesting insight is that location does not depend on biorefinery size due to a high effort in optimizing supply chain tactic decisions.

Total costs information is showed in Figure 9 where costs are divided into biomass costs, transportation costs and storage costs. All numbers are available upon request to the authors. Note that in the intermediate-collector strategy storage cost includes the cost of setting up the 
intermediate facilities. Intermediate-collector alternative is always a better choice in terms of costs. The lower depreciation rate as well as the flexibility of having intermediate warehouses allows reducing significantly the purchase invoice. On average, a reduction of $11 \%$ can be found in biomass costs. On the other hand, transportation costs and storage cost are much higher $(41 \%$ and $49 \%$ higher, respectively) because more distance is driven as well as the additional cost of setting up the intermediate-collectors. As result, total reduction costs account for $2.68 \%$, on average. Direct supply strategy is preferred in Scenario 13 (350,000 size and 1 exploitation factor), thought. An explanation could be that sufficient biomass is extended around location 20 that make it the direct supply a better choice. On the other hand, a 5\% reduction costs is found in Strategy 5 due to the different biorefinery location and the high biomass availabilities as exploitation factor is set at 1.5 .

A comparison between distances driven is given in Figure 8. As expected, 30\% more distance is driven in the intermediate-collector strategy. As a result, in the direct supply strategy just 5.8 kilometers are driven for every ton required and 8.2 in the intermediate-collector one instead.

Figure 7 Optimal location for the biorefinery based on consumption and exploitation factor

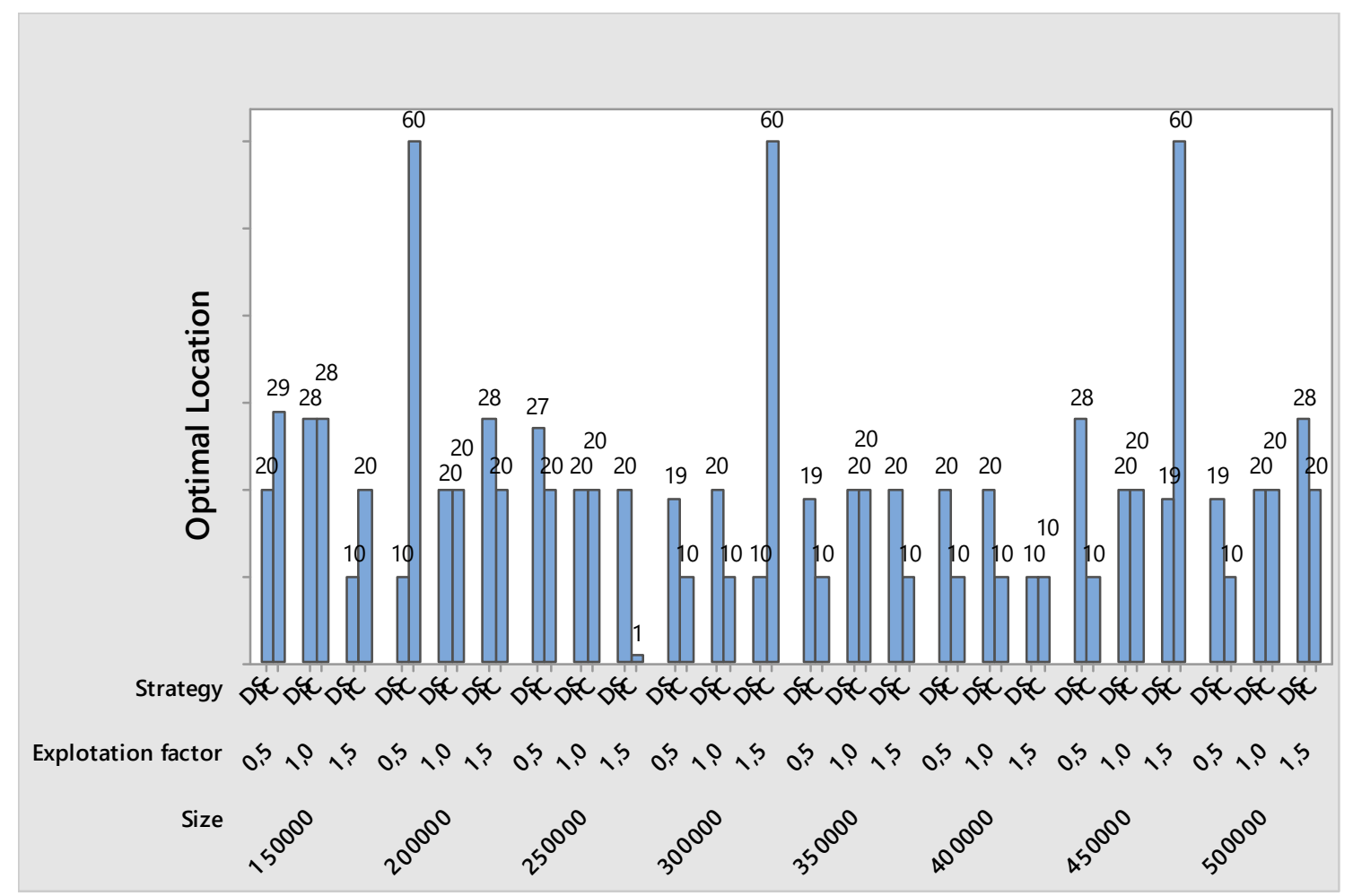


Figure 8 Distance driven comparison based on consumption and exploitation factor

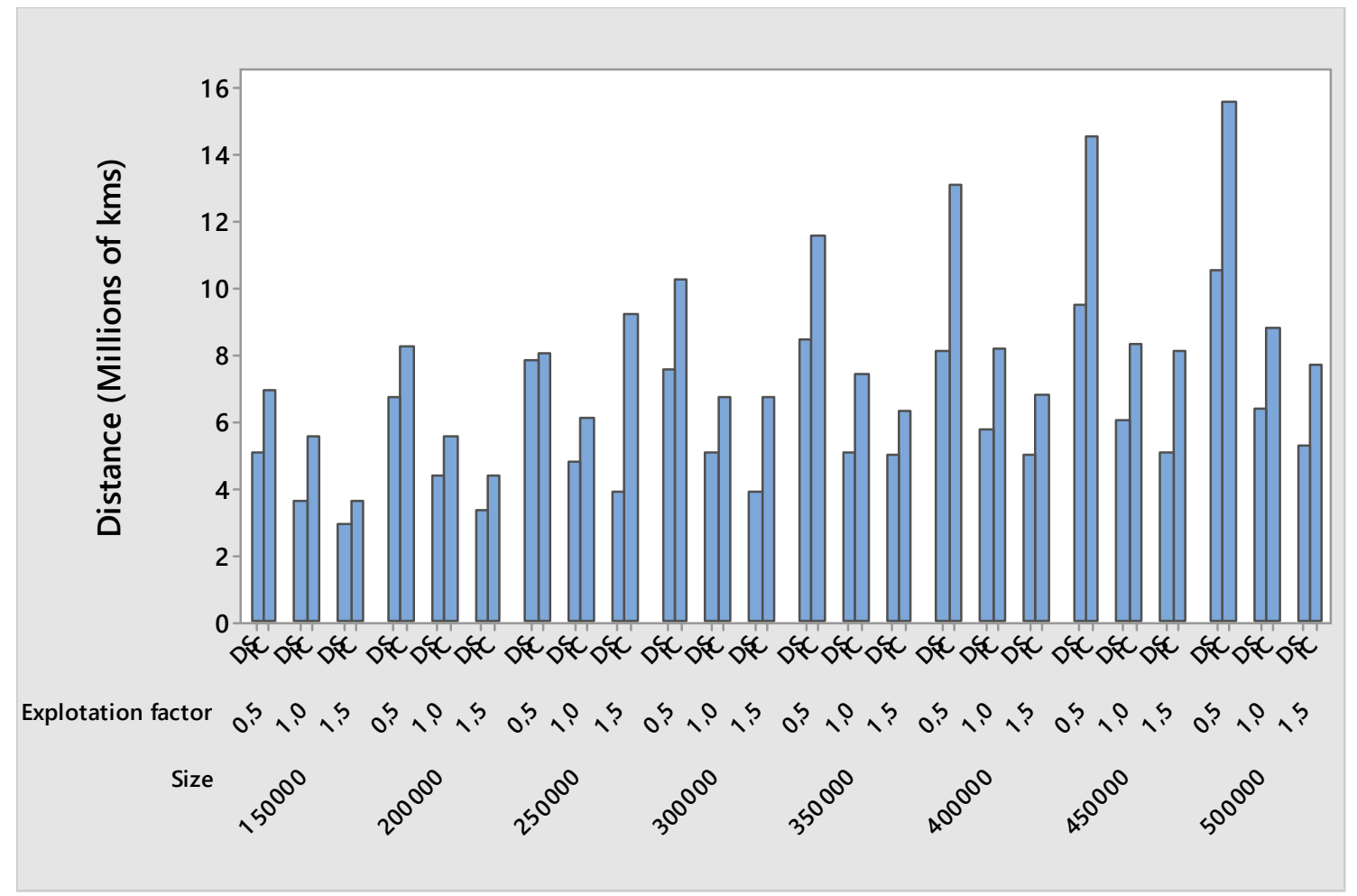

Figure 9 Total cost comparison based on size and exploitation factor

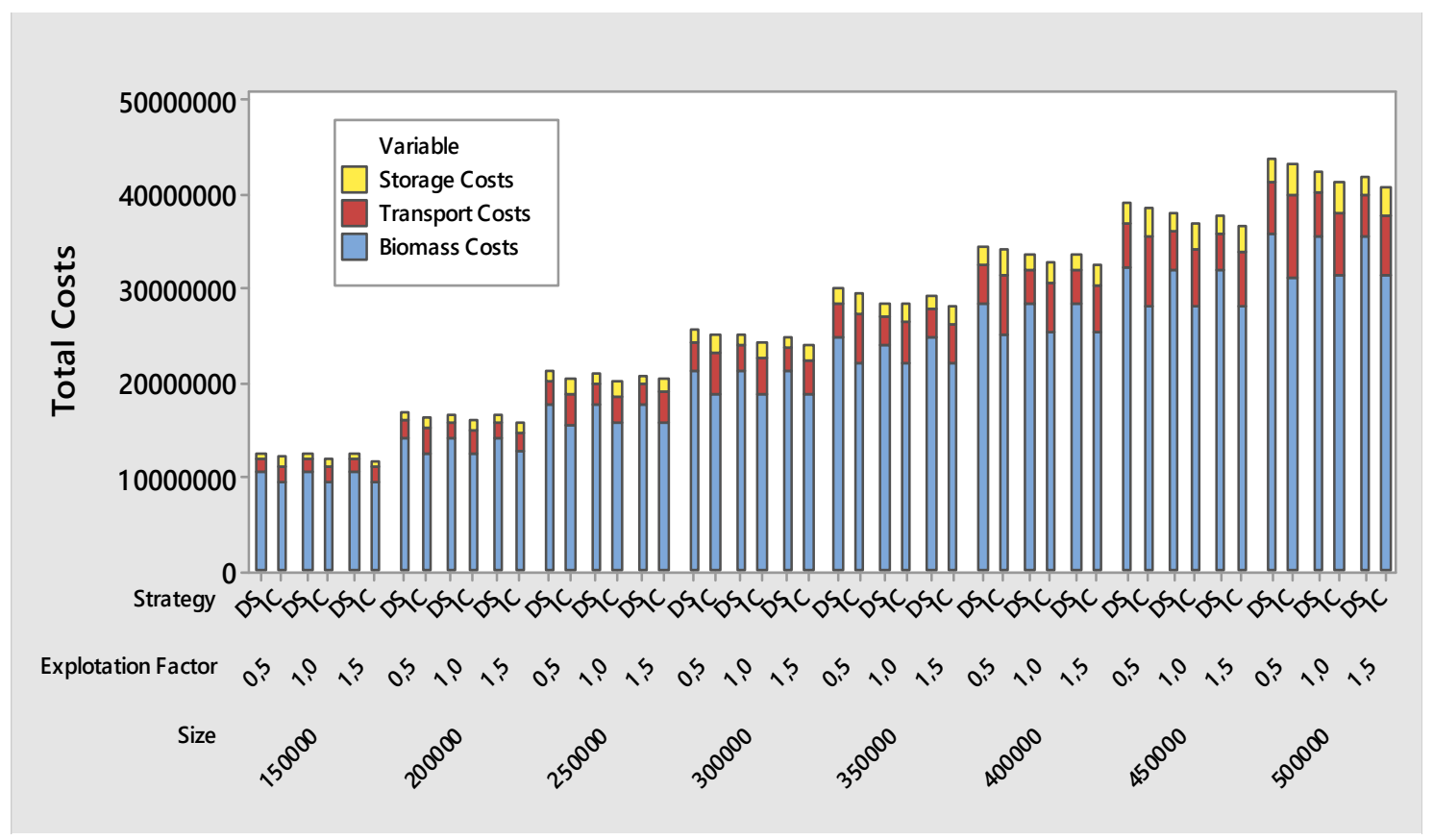

\section{Conclusions}

Facility location problems deal with strategic decisions. They are made at the top management level of the company since their effects may compromise the development of the firm and even 
its own survival. Additionally, forthcoming tactical and operational decisions will depend on the previous strategic ones. For that reason, thoughtful analyses are required in order to evaluate properly their potential effects. Strategic Policy Evaluation aims to help decision makers in their strategic decisions by evaluating them among several scenarios.

A case study, in which a biorefinery has to be sited, is investigated in the regions of Navarre, La Rioja and Aragon (Northern Spain). Tactical decisions ranging from purchase policy, transport policy and storage policy are then carried out. Only local and limited biomass (winter cereal straw, corn straw, alfalfa, rape straw, and rice straw) can be harvested for feeding the biorefinery and two different and mutually exclusive storage strategies were assessed (1) direct supply from crops to biorefinery and (2) intermediate-collectors. Additionally, exploitation factors (the proportion of the total biomass available that effectively could be used for feeding a biorefinery) and biorefinery size (measured as biomass consumption) were used to generate several scenarios in which the strategic decision of location and all the tactic decisions must be taken.

According to the results, biorefinery location should be sited in northwest study area as most of the potential locations obtained correspond to that area (see, for instance, PL10, PL20 or PL28). In this sense, the Figure 10 shows the solution corresponding to Scenario 7. In this case, intermediate-collectors are set up in potential locations number 20 and 6. In Figure 10, crops fields are painted in the same color the intermediate-collector/biorefinery they are serving. Moreover, there are some other crops fields that are not used.

Consequences of locating the biorefinery outside the "optimal area" can be computed. For instance, a wrongly number 75 location, in Southeast study area, would increase total cost by $15 \%$. Once the location is fixed, significant differences arise between direct supply and intermediate-collector alternatives. The lower depreciation rates as well as the higher flexibility of having intermediate-collectors, make that alternative preferred over the direct supply strategy. Differences in terms of costs may rise up to $5 \%$ which represents about $€ 2.5$ million yearly. Kilometers driven are significantly higher (about 30\%) in the intermediate-collector alternative. This may incite a higher environmental impact that should be taken into account. The increasing concerns about environmental issues as well as the appearance of new environmental-taxes may compensate the savings of intermediate-collector alternative. If a green scenario had been contemplated, direct supply alternative would have been preferred and another location selected. Internal purchase policy, transportation policy and storage policy can be analyzed within the scenarios. Thus, it is provided key information about critical biomass, crops and times. Therefore, decision makers could take advance in next negotiation processes with farmers. Moreover, a deeper transportation analysis can be performed pointing the optimal vehicle fleet combination 
(large, medium and small). Finally, the storage management is critical in that context. Information about stock levels over the year can be easily filter from the results.

Figure 10 Solution obtained when solving scenario 7

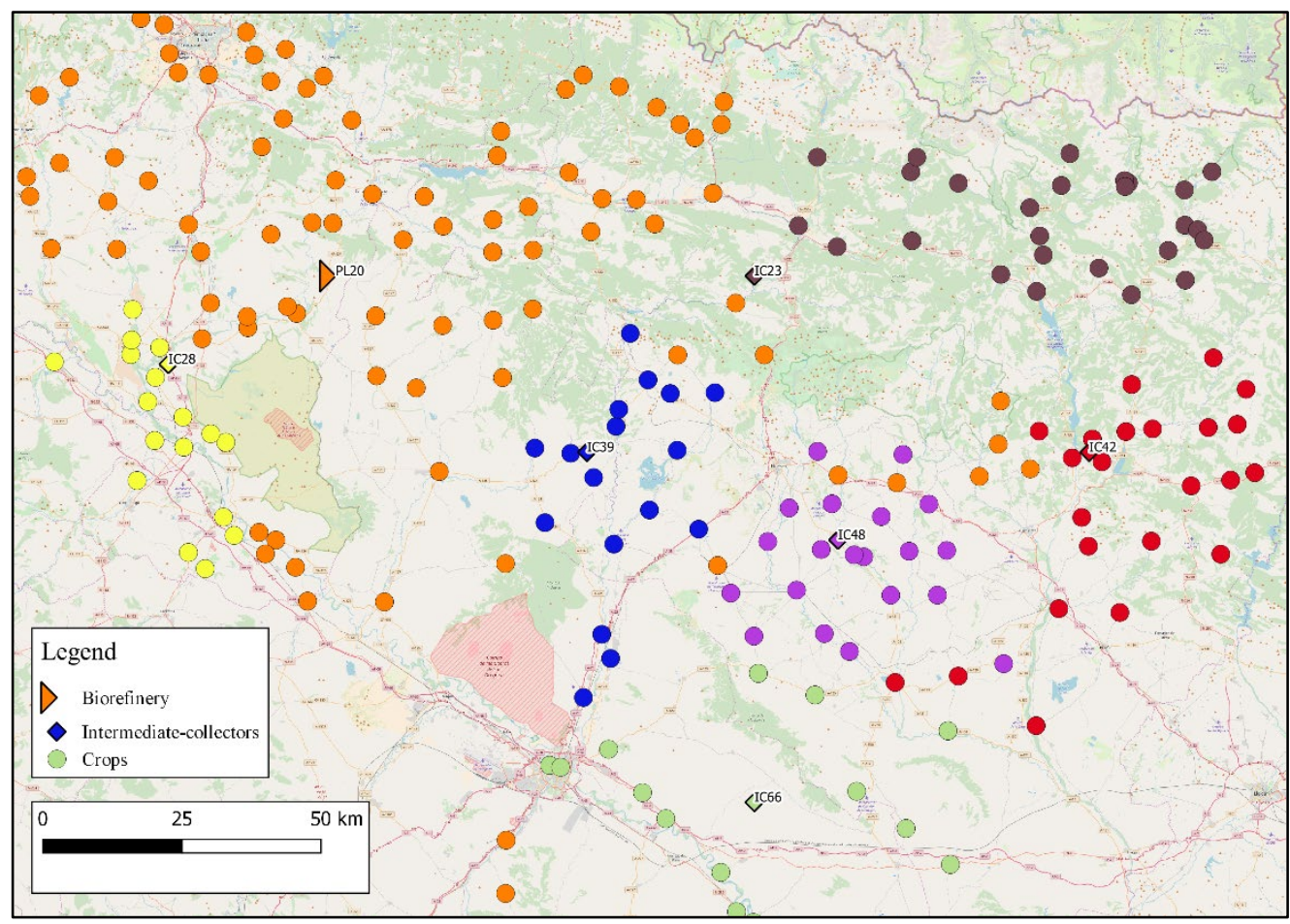

\section{Acknowledgments}

This work has been partially supported by the Government of Navarre (Biorefinery Navarra project IIM14196.RI1) the Spanish Ministry of Economy and Competitiveness (TRA201348180-C3-P and TRA2015-71883-REDT), and the Ibero-American Program for Science and Technology for Development (CYTED2014-515RT0489). Likewise, we want to acknowledge the support received by the CAN Foundation in Navarre, Spain (Grant CAN2017-6101).

\section{References}

Aresta, M., Dibenedetto, A., and Dumeignil, F. (2012). Biorefinery: from biomass to chemicals and fuels. Walter de Gruyter, Berlin.

Ayodele, T., Ogunjuyigbe, A., and Amusan, T. (2016). Wind power utilization assessment and economic analysis of wind turbines across fifteen locations in the six geographical zones of Nigeria. Journal of Cleaner Production, 129: 341-349.

Chen, L., Olhager, J., and Tang, O. (2014). Manufacturing facility location and sustainability: A literature review and research agenda. International Journal of Production Economics, 149:154-163 
Delivand, M., Cammerino, A., Garofalo, P., and Monteleone, M. (2015). Optimal locations of bioenergy facilities, biomass spatial availability, logistics costs and GHG (greenhouse gas) emissions: a case study on electricity productions in South Italy. Journal of Cleaner Production, 99:129-139.

Department of Agriculture of Aragon (2016) Encuesta Agraria URL: http://www.aragon.es/DepartamentosOrganismosPublicos/Departamentos/DesarrolloRuralSostenibilid ad/AreasTematicas/EstadisticasAgrarias/ci.ESTADISTICAS AGRICOLAS.detalleDepartamento?cha nnelSelected=1cfbc8548b73a210VgnVCM100000450a15acRCRD (In Spanish). Lasts Access: December, $19^{\text {th }} 2017$

Department of Agriculture of La Rioja (2016) Encuesta Agraria URL: http://www.larioja.org/agricultura/es/estadistica-agraria (In Spanish). Lasts Access: December, 19 2017

Department of Agriculture of Navarre (2016) Encuesta Agraria URL: http://www.navarra.es/home es/Temas/Ambito+rural/Vida+rural/Observatorio+agrario/Agricola/Infor

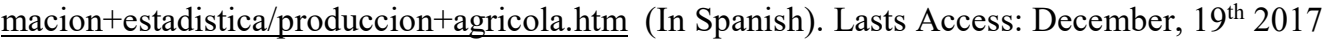

European Environment Agency (2015). Energy efficiency and energy consumption in the transport sector. Technical Report.

Haque, M., Epplin, F., Biermacher, J., Holcomb, B., and Kenkel, P. (2014). Marginal cost of delivering switchgrass feedstock and producing cellulosic ethanol at multiple biorefineries. Biomass and Bioenergy, 66:308-319.

Lado-Sestayo, R., Vivel-Búa, M., and Otero-González, L. (2016). Survival in the lodging sector: An analysis at the firm and location levels. International Journal of Hospitality Management, 59: 19-30.

Luo, L., Voet, E. and Huppes, G. (2010) Biorefining of lignocellulosic feedstock - Technical, economic and environmental considerations. Bioresource Technology, 101(13): 5023-5032.

Marvin, W., Schmidt, L.D. and Daoutidis, P. (2013). Biorefinery location and technology selection through supply chain organization. Industrial and Engineering Chemistry Research, 52:3192-3208

Masiero, L., Heo, C. Y., and Pan, B. (2015). Determining guests' willingness to pay for hotel room attributes with a discrete choice model. International Journal of Hospitality Management, 49: 117-124.

Mele, F., Guillén-Gosálbez, G., and Jiménez, L. (2009). Optimal planning of supply chains for bioethanol and sugar production with economic and environmental concerns. Computer Aided Chemical Engineering, 26: 997-1002.

Melo, M., Nickel, S., and Saldanha-da-Gama, F. (2009). Facility location and supply chain managementA review. European Journal of Operational Research, 196(2): 401-412.

Panichelli, L., and Gnansounou, E. (2008). GIS-based approach for defining bioenergy facilities location: A case study in Northern Spain based on marginal delivery costs and resources competition between facilities. Biomass and Bioenergy, 32(4): 289-300

Papadakis, V., and Barwise, P. (2012). Strategic decisions. Springer Science and Business Media, Boston. San Miguel, G., Corona, B., Ruiz, D., Landholm, D., Laina, R., Tolosana, E., and Cañellas, I. (2015). Environmental, energy and economic analysis of a biomass supply chain based on a poplar short rotation coppice in Spain. Journal of Cleaner Production, 94: 93-101. 
Serrano-Hernandez, A., Faulin, J., Astiz, P., Sánchez, M., and Belloso, J. (2015). Locating and Designing a Biorefinery Supply Chain under Uncertainty in Navarre: A Stochastic Facility Location Problem Case. Transportation Research Procedia, 10:704-713.

Serrano-Hernandez, A., Faulin, J., Pintor, J.M. and Belloso, J. (2017). Determining an Optimal Area to Locate a Biorefinery under Economic and Environmental Criteria. Transportation Research Procedia, 22: 95-104.

Spanish Government (2017). Observatorio de costes del transporte de mercancías de carretera. Technical Report. URL: https:/www.fomento.gob.es/NR/rdonlyres/CD6BCD8F-0642-40D0-A6FCB47C2C1E88FD/144017/ObservatorioMercanc\%C3\%ADasjulio2017.pdf (In Spanish). Lasts Access: December, $19^{\text {th }} 2017$.

Spigarelli, F., and Lv, P. (2016). The determinants of location choice: Chinese foreign direct investments in the European renewable energy sector. International Journal of Emerging Markets, 11(3):333-356

Xie, Y. Zhao, K. and Hemingway, S. (2009). Optimally locating biorefineries: A GIS-Based Mixed Integer Linear Programming Approach. 51st Transportation Research Forum, 1:540-561

Yang, Y., Luo, H., and Law, R. (2014). Theoretical, empirical, and operational models in hotel location research. International Journal of Hospitality Management, 36: 209-220.

You, F., and Wang, B. (2011). Life cycle optimization of biomass-to-liquids supply chains with distributed - centralized processing networks. Industrial Engineering Chemical Research, 50:10102-10127.

You, F., Tao, L., Graziano, D., and Snyder, S. (2012). Optimal design of sustainable cellulosic biofuel supply chains: Multiobjective optimization coupled with life cycle assessment and input-output analysis. AIChE Journal, 58:1157-1180.

Yu, T., He, L., English, B., and Larson, J. (2014). GIS-based optimization for advanced biofuels supply chains: a case study in Tennessee. Lecture Notes in Management Science, 6:217-227

Zanjirani, R., and Hekmatfar, M. (2009) Facility Location Concepts, Models, Algorithms and Case Studies. Physica Verlag, Heidelberg. 